\title{
两种沉水植物黑藻和伊乐藻的种间竞争
}

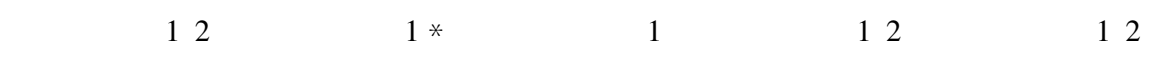 \\ （1 中国科学院武汉植物园水生植物生物学实验室, 武汉 430074) (2 中国科学院研究生院 北京 100049)
}

摘 要 采用取代系列实验方法, 主要从竞争期的长短出发, 研究了黑藻 (Hydrilla verticillata) 和伊乐藻 ( Elodea nuttallii) 的种间竞争关系, 并考查了在不同底质(土壤)肥力下两者种间竞争能力的变化情况。实验发现, 伊乐藻由于 具有较强的耐寒能力, 在冬春时空竞争方面占有明显的优势, 从而在周年实验中表现出较强的竞争优势 取代黑藻 生长。而在短期实验中, 黑藻由于可在水面生长形成较上位的冠层的特性, 与伊乐藻相比在水体上层空间占领和 阳光获取方面具有一定的优势, 因此造成两种间竞争的不平衡, 竞争偏利于黑藻, 且这种优势随底质(土壤)肥力的 增加而有所增强, 但并没有明显取代现象的发生, 两物种可以在混合种群中共存。

关键词 伊乐藻 黑藻 竞争 取代系列实验 沉水植物 土壤肥力

\section{INTER-SPECIFIC COMPETITION BETWEEN TWO SUBMERGED MACROPHYTES , ELODEA NUTTALLII AND HYDRILLA VERTICILLATA}

\author{
XU Jing-Wei ${ }^{1}{ }^{2}$, LI Wei $^{1 *}$, LIU Gui-Hua ${ }^{1}$, ZHANG Li-Jing ${ }^{1}{ }^{2}$, and LIU Wen-Zhi ${ }^{1}{ }^{2}$ \\ ${ }^{1}$ Laboratory of Aquatic Plant Biology, Wuhan Botanical Garden , Chinese Academy of Sciences, Wuhan 430074 , China , and ${ }^{2}$ Graduate University \\ of Chinese Academy of Sciences , Beïjng 100049 , China
}

\begin{abstract}
Aims Elodea nuttallii and Hydrilla verticillata have similar morphology. E. nuttallii is an alien aquatic plant introduced into China in 1980s. Both species occupy similar habitat ; therefore , they are potential competitors. We investigated :1) weather inter-specific competition occurred ,2) which species had superior competitive abilities, and 3) what factors influenced competitive results.

Methods Two replacement series experiments were carried out to analyze interspecific competition between $E$. nuttallii and $H$. verticillata. In replacement series experiments, the planting densities of the two species were varied but the total density was held constant. The first experiment assessed competitive ability at two levels of soil fertility, and the second investigated the long-term competitive growth of $E$. nuttallii and $H$. verticillata.

Important findings $E$. nuttallii had superior cold resistance and advantages in competing for space-time during winter and spring, so it grew well in the long-term experiment and eventually replaced $H$. verticillata . In the short-term experiment , $H$. verticillata formed an upper canopy, giving it advantages in competing for light and occupying the water surface, causing imbalanced competition between $H$. verticillata and $E$. nuttallii. H. verticillata was the stronger competitor at both levels of soil fertility, and increased soil fertility could strengthen its competitive abilities ; however, it could not replace $E$. nuttallii and they could coexist in the mixed population. This study suggests competitive abilities are integrated abilities of plants and that morphological traits , life history traits and environmental factors could affect the results of competition. Therefore, investigations of plant interactions should encompass a wide array of factors.
\end{abstract}

Key words Elodea nuttallii , Hydrilla verticillata , competition, replacement series experiment, submerged macrophyte , soil fertility

竞争是植物界普遍存在的现象, 早在 19 世纪 Darwin 就已经认识到竞争在有机体自然选择过程中 的中心地位。自此, 竞争一直被看作塑造植物形态、 生理、生活史特征和植物群落结构、动态的主要动力
之一。在植物生态学中, 为了认识植被的演替类型, 植物群落的多样性和稳定性, 寻求半自然生态系统 的最适管理对策,科学家们开始研究植物间的竞争 现象 (Arassen, 1983; ;ilman, 1988;Grime, 2001)。 
伊乐藻 (Elodea nuttallii) 和黑藻 (Hydrilla verticillata) 同属于水鳖科 (Hydrocharitaceae), 草本沉水植 物 营养体形态(如分枝类型、叶型、不定根的产生 等)和生长方式(如形成冠层等)极为相似, 都具有较 强的环境适应能力, 能在湖泊、河流、沟渠、水田等多 种生境存活, 并依靠其强势的断枝无性繁殖能力迅 速扩展种群 (连光华和张圣照, 1996;Barrat-Segretain et al. ,2002)。其中伊乐藻为一外来物种, 原产美 洲 20 世纪 80 年代经由日本引入我国，具有较大的 经济利用价值，在渔业生产中作为优质的饵料，并在 水环境修复工程中被大量利用 (朱松泉和刘正文, 1996 ;高光, 1996 ;朱伟等, 2004)。

伊乐藻具有极强的入侵性, 19、20世纪被引入 欧洲及其它国家，迅速成为优势种群，在法国、日本、 瑞士、不列颠群岛等国家和地区一些受干扰较大的 水体中很快取代其它物种, 成为当地的优势种 (Kunii , 1984 ; Barrat-Segretain , 2001 ; Barrat-Segretain et $a l$ ，，2002）。目前在我国某些湖区也已经形成大面 积的沉水植被, 如张圣照等 (1999) 在对东太湖水生 植被的调查研究中就发现, 伊乐藻已经开始侵入当 地的优势群丛中,并形成一定的规模。谷孝鸿等 (2005) 再次对东太湖水生植被的调查中也发现， 1981 年东太湖沉水植物群落主要以黑藻、竹叶眼子 菜 (Potamogeton malainus)、苦草 (Vallisneria spiralis) 为优势建群种, 1986 年引进伊乐藻后, 1996 年主要 以微齿眼子菜 (Potamogeton maackianus) 为优势建群 种, 伊乐藻成为亚优势种, 2002 年主要以伊乐藻和 无根植物金鱼藻 (Ceratophyllum demersum) 为优势建 群种。由此可见, 伊乐藻作为一外来物种, 已经对水 生生态环境尤其是物种多样性造成了一定的影响。 近年来, 伊乐藻作为一种对富营养化耐受性较强的 沉水植物在许多退化水域生态系统重建工作中, 被 人为地引进和扩增, 尽管取得了一定的水质改善效 果，但是对其潜在的生态效应尚缺乏科学的研究。 本文研究了伊乐藻与黑藻之间的竞争关系, 以期为 伊乐藻对乡土种的影响提供基础资料。

植物的种间竞争可以通过不同的实验方法进行 研究取决于试验者的研究目的。其中取代系列实 验设计可能是生态学中应用最为广泛的一种研究生 物间相互作用的方法 (Cousens, 1991;Gibson et al. , 1999)。在取代系列实验 (Replacement series experiment 或 Substitutive series experiment)中, 两物种的总 密度保持恒定, 而各自在混生种群中的密度比在 0 1之间变化 (Cousens , 1991;Gibson et al . 1999 ;Jol- liffe,2000 李博,2001) ,它具有以下优点: 可以较好 的研究两物种的种间关系, 找出哪个具有更强的竞 争能力; 比较不同条件下的竞争结果, 研究生态位的 分化 探讨物种多样性和群落生产力的关系, 确定生 物间相互作用的性质和类型; 研究环境资源的利用 效率等等 $(\mathrm{Li} \&$ Hara，1999; Jolliffe,2000; 李博, 2001)。

本实验采用取代系列实验设计方法, 主要从竞 争期的长短出发, 研究了伊乐藻和黑藻的种间竞争 关系, 以及在不同基质营养条件下竞争能力的变化, 探讨了伊乐藻和黑藻的竞争能力以及影响竞争能力 的物种生物学特性, 为沉水植物竞争研究提供一定 的参考, 并为评价伊乐藻作为外来种的生态影响, 为 沉水植物群落管理以及沉水植物的合理开发利用提 供基础资料。

\section{1 材料和方法}

\section{1 伊乐藻、黑藻生物学特征简介}

伊乐藻,自然条件下常常表现出以年为周期的 生长发育节律, 早春萌发, 夏季高温期生长停滞, 进 入休眠状态; 秋季再度生长, 形成新的种群 (Kunii， 1982 ;Best \& Dassen,1987 ;胡耀辉,1996)。它具有较 宽的温度适应范围, 水温 $5 \sim 30{ }^{\circ} \mathrm{C}$ 都能处于正常的 营养生长状态, 能够忍受 $0{ }^{\circ} \mathrm{C}$ 甚至冰点以下的寒冷, 最适温度 $25{ }^{\circ} \mathrm{C}$ 左右, 30 ${ }^{\circ} \mathrm{C}$ 以上对其生长有抑制, 6 月下旬或 7 月上旬达到最大生物量 (Kunii, 1984; 朱 松泉和刘正文, 1996)。在温度适宜的地区, 一般水 温高于 $4 \pm 1{ }^{\circ} \mathrm{C}$ 时 植株可以继续保持活力状态正常 生长, 但是生长速率和生物量积累较小, 在人工栽培 管理条件下,营养体可以经年生长 (Kunii ,1982， 1984 ;Best \& Dassen ,1987)。

黑藻, 全年生长发育期 $250 \mathrm{~d}$ 左右,喜温耐热, 适应性强, 生长迅速, 能以断枝、冬芽、种子等多种方 式繁殖。一般在 4 月中上旬, 黑藻冬芽经过休眠萌 发新枝, 生长旺盛期间, 上部可漂浮于水面生长, 形 成茂密的冠层, 夏季水温高于 $40{ }^{\circ} \mathrm{C}$ 时生长减慢, 10 月达到最大生物量 秋末冬初, 直立茎的小枝顶端形 成长卵圆形的冬芽, 脱离母株后, 沉入水底过冬, 翌 年春季萌发成为新的植株, 11 月下旬植株茎叶变黄 并陆续死亡(文明等, 1994,1995)。黑藻具有较强的 无性繁殖和定居能力, 对环境的适应能力较强(中国 科学院武汉植物研究所, 1983;陈洪达, 1989; 崔心红 等 ,2000)。 
竞争结果的解释很大程度上依赖于采用什么样 的测度方法 (Freckleton \& Watkinson ,1999) , 在众多植 物性状与竞争能力的关系上, 产量或者说生物量是 竞争能力最好的估计量 (Gaudet \& Keddy ,1988) ,通 过生物量的变化来反映植物的竞争能力，绝大多数
的竞争指标 (Competition indices) 都是在生物量的基 础上衍生出来的。根据前人关于取代系列实验竞争 指标的论述 (Jolliffe 2000; Williams \& McCarthy,2001; Weigelt \& Jolliffe, 2003) ,以下指标可以较好的测度两 物种竞争能力 (表 1)。

表 1 竞争能力测度指标

Table 1 Indices to quantify the ability of competition

\begin{tabular}{|c|c|c|c|}
\hline 指标名称 Name of indices & 缩写 Abbreviation & 公式 Formulae & 来源 Source \\
\hline 相对产量 & $R Y$ & $R Y_{\mathrm{a}}=Y_{\mathrm{ab}} /\left(p_{\mathrm{a}} Y_{\mathrm{a}}\right)$ & Fowler(1982) \\
\hline Relative yield & & $R Y_{\mathrm{b}}=Y_{\mathrm{ba}} /\left(p_{\mathrm{b}} Y_{\mathrm{b}}\right)$ & \\
\hline 相对产量总和 & $R Y T_{\mathrm{ab}}$ & $R Y T_{\mathrm{ab}}=R Y_{\mathrm{a}}+R Y_{\mathrm{b}}$ & de Wit(1960) ; de Wit \\
\hline Relative yield total & & & \& van den Bergh(1965) \\
\hline 相对竞争强度 & $R C I^{*}$ & $R C I_{\mathrm{a}}=\left(p_{\mathrm{a}} Y_{\mathrm{a}}-Y_{\mathrm{ab}}\right) / p_{\mathrm{a}} Y_{\mathrm{a}}=$ & Grace(1995) \\
\hline Relative competitive intensity & & $1-R Y_{\mathrm{a}}$ & \\
\hline 竞争攻击力 & $A G$ & $A G=0.5\left(R Y_{\mathrm{a}}-R Y_{\mathrm{b}}\right)$ & McGilchrist \& Trenbath(1971) \\
\hline Aggressivity & & & \\
\hline 相对拥挤系数 & $R C C^{*}$ & $R C C_{\mathrm{a}}=p_{\mathrm{b}} Y_{\mathrm{ab}} / p_{\mathrm{a}}\left(Y_{\mathrm{a}}-Y_{\mathrm{ab}}\right)$ & Firbank \& Watkinson( 1985) \\
\hline Relative crowding coefficient & & & \\
\hline
\end{tabular}

此外，取代系列实验图表 (Replacement diagram) 由于其直观性和便利性也经常被用来解释取代系列 实验结果( Firbank \& Watkinson,1985; Jolliffe, 2000; 李 博 2001），在图表中将物种实测生物量与预期生物 量绘制在一起, 比较其差异，从而判断竞争是否发生 以及竞争的类型, 并结合产量-密度反应模型 (Yielddensity response models) (Watkinson, 1981 ; Wright, 1981 ;Spitters ,1983 ; Turkington \& Jolliffe,1996 ; Baumann et al. 2001) 来分析物种的竞争能力。产量-密 度反应模型经常被用来分析物种单位面积产量与组 成种密度的关系或个体产量与组成种密度的关系， 对于两组成种的实验 (以本实验伊乐藻、黑藻为例), 其方程如下：

$$
\begin{aligned}
& Y \mathrm{e}=N_{\mathrm{e}} /\left(A_{\mathrm{e} 0}+A_{\mathrm{ee}} N_{\mathrm{e}}+A_{\mathrm{eh}} N_{\mathrm{h}}\right) \\
& Y_{\mathrm{h}}=N_{\mathrm{h}} /\left(A_{\mathrm{h} 0}+A_{\mathrm{hh}} N_{\mathrm{h}}+A_{\mathrm{he}} N_{\mathrm{e}}\right)
\end{aligned}
$$

其中 $Y_{\mathrm{e}}$ 和 $Y_{\mathrm{h}}$ 表示每个塑料盒中的生物量干 重; $N_{\mathrm{e}} 、 N_{\mathrm{h}}$ 代表伊乐藻、黑藻植株数目; ;常数项 $A_{\mathrm{e} 0}$ 和 $A_{\mathrm{h} 0}$ 用来估计在单独种植时最大产量的倒数; $A_{\mathrm{ee}}$ 和 $A_{\mathrm{hh}}$ 用来估计种内竞争 称为种内竞争系数; $A_{\mathrm{eh}}$ 和 $A_{\mathrm{he}}$ 用来估计种间竞争, 称为种间竞争系数; $A_{\mathrm{ee}} / A_{\mathrm{eh}}$ 和 $A_{\mathrm{hh}} / A_{\mathrm{he}}$ 用来估计种内竞争、种间竞争的权重。

\section{3 实验设计}

\section{3 .1 短期实验}

实验选择在两物种初春开始生长至夏季高温生 长减缓期间进行,此段时间均为两物种的旺盛生长 期。剪取大小一致的不带分枝的伊乐藻、黑藻枝尖 部分 $20 \mathrm{~cm}$ 。种植容器为 $45 \mathrm{~cm}$ (长) $\times 32 \mathrm{~cm}$ (宽) $\times$ $16 \mathrm{~cm}$ (高)的塑料盒子, 内装 $8 \mathrm{~cm}$ 深的底质(约 7.5 $\mathrm{kg}$ ), 两种营养水平的底质分别为黄壤土 (总氮： $0.576 \pm 0.023 \mathrm{mg} \mathrm{g}^{-1}$,总磷: $0.223 \pm 0.012 \mathrm{mg} \mathrm{g}^{-1}$ ) 和添加了 $40 \mathrm{~g}$ 缓释肥 (奥绿肥 5 号 Osmocote, 德国, 规格 Osmocote14-13-13 肥力有效期土壤平均温度 16 ${ }^{\circ} \mathrm{C} 6 \sim 7$ 个月、 $21{ }^{\circ} \mathrm{C} 5 \sim 6$ 个月、 $26{ }^{\circ} \mathrm{C} 3 \sim 4$ 个月、 31 ${ }^{\circ} \mathrm{C} 2.5 \sim 3$ 个月) 的黄壤土 (总氮: $1.323 \pm 0.023 \mathrm{mg}$ $\mathrm{g}^{-1}$,总磷 $0.916 \pm 0.012 \mathrm{mg} \mathrm{g}^{-1}$ ) ,肥料放置在盒底， 缓慢释放。总密度设置为每盒 20 株 (约 140 株. $\left.\mathrm{m}^{-2}\right)$, 密度比设置为 $20: 0 、 15: 5 、 10: 10 、 5: 15 、 0: 20$ ， 每个密度 4 个重复。插植剪取的伊乐藻、黑藻于底 质中, 插入约 $2 \mathrm{~cm}$, 使之固定。分开放置于武汉植 物园水生植物温室水泥池中 $(2 \mathrm{~m}$ 长 $\times 2 \mathrm{~m}$ 宽 $\times 1 \mathrm{~m}$ 深), 每个池中放一个密度处理 (4 盒), 间隔均匀, 横 纵间隔约 $40 \mathrm{~cm}$, 使之互不干扰。武汉东湖后湖湖水 培养, 湖水在进入温室使用前通过人工池塘(布满水 草) 的净化。水深设置为 $1 \mathrm{~m}$, 湖水总氮 $1.161 \pm$ $0.157 \mathrm{mg} \mathrm{L}^{-1}$ 、总磷 $0.038 \pm 0.003 \mathrm{mg} \mathrm{L}^{-1}$, 每两星 期换水一次, 以消除底质营养释放对水质的影响。 
实验从 2005 年 4 月 30 号开始, 周期为 10 个星期, 至 7 月 11 号结束。将所有处理取出, 分开地上、地下部 分,风干, 在 $80{ }^{\circ} \mathrm{C}$ 烘箱中烘至恒重得其生物量干重。

\subsection{2 周年竞争态势实验}

剪取大小一致不带分枝的伊乐藻、黑藻枝尖部 分约 $20 \mathrm{~cm}$ 。实验容器为开口内径 $64 \mathrm{~cm}$, 深 $60 \mathrm{~cm}$ 的水缸, 内装填 $10 \mathrm{~cm}$ 深的底泥, 底泥为采自武汉植 物园水生植物资源固旁的池塘淤泥 (总氮: $1.672 \pm$ $0.315 \mathrm{mg} \mathrm{g}^{-1}$,总磷 : $\left.1.225 \pm 0.243 \mathrm{mg} \mathrm{g}^{-1}\right)$ 。按照 取代系列实验方法, 每缸种植 30 株, 密度比设置为 30:0, 25:5, 20:10, 15:15, 10:20,5:25, 0:30, 每个密 度 6 个重复。插植剪取的伊乐藻、黑藻于底泥中约 $2 \mathrm{~cm}$, 使之固定。采用武汉东湖后湖湖水培养, 湖水 灌满水缸, 水体总氮 $1.824 \pm 0.106 \mathrm{mg} \mathrm{L}^{-1}$,总磷 $0.021 \pm 0.007 \mathrm{mg} \mathrm{L}^{-1}$ 。每个星期加水, 以补充湖水 蒸发损失。定期观察记录其生长情况, 考查其最终 的竞争格局。实验周期为 2004 年 9 月至 2005 年 9 月,此时两物种皆完成了周年生长。

\section{4 数据分析}

利用 Excel 和 SPSS12.0 数据分析软件对数据进 行统计分析。不同处理间的差异性采用双因素和多 因素方差分析, 产量-密度方程采用 SPSS12.0 中的 非线性回归分析方法 综合所有密度比例下伊乐藻、 黑藻生物量干重, 进行非线性回归, 用连续二次方程 迭代法求解拟合系数 构建方程。

\section{2 结果与分析}

\section{1 短期实验}

\section{1 .1 伊乐藻、黑藻生长状况}

伊乐藻、黑藻断枝插植 $3 \mathrm{~d}$ 后均生出白色不定 根，成活率达到 $100 \% 5 \mathrm{~d}$ 后即有新枝萌生，30 d 左 右黑藻即可生长至水面，此后在近水面处产生大量 分枝, 浮在水面蔓延生长, 形成茂密的冠层, 而伊乐 藻生长稍慢, 植株未能伸长至近水面, 分枝纤细柔弱 易倒伏，在水体中下层形成地毯状冠层，占据近底质 处的空间。实验结束时, 由于水温的持续升高伊乐 藻达到最大生长后, 生长减缓或停滞, 黑藻生长亦显 著减慢, 新生分枝数明显减少。

图 1 所示实验期间两种底质下伊乐藻、黑藻不 同密度组合的每盆生物量干重情况。与低肥力相 比, 高肥力下黑藻的干重增加至 1.3 倍, 伊乐藻增加 至 1.4 倍, 两物种均有所增加。黑藻植株生长速率 明显快于伊乐藻 $(p<0.05$, 独立样本 $t$ 检验) ,且其 枝条可以生长至水面在水面漂浮生长, 占据水体表
面广阔水体，此外黑藻的根系较伊乐藻发达，黑藻比 伊乐藻分配较多的生物量到根部(表 2)。

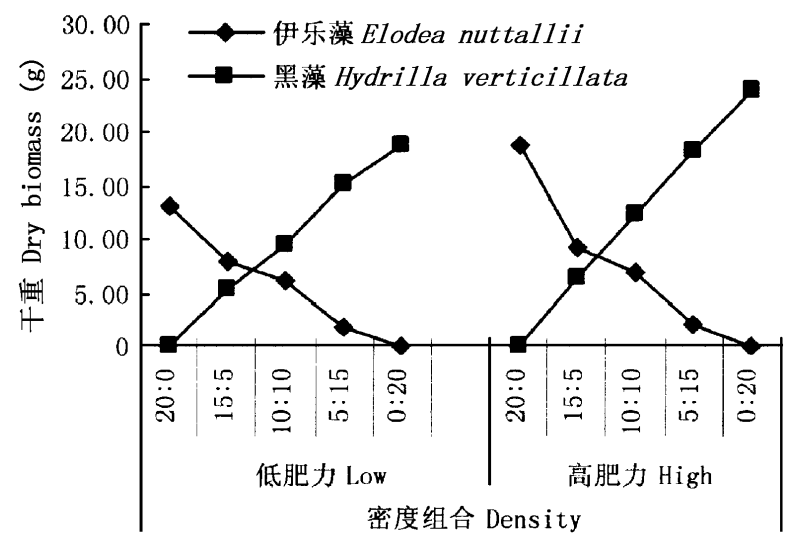

图 1 伊乐藻、黑藻生物量干重

Fig. 1 Dry biomass of Elodea nuttallii and Hydrilla verticillata at two levels of soil fertility

\subsection{2 竞争量化指标}

根据单种及混合种植所得两物种的生物量干 重 计算各竞争指标的值 结果如表 3 所示：

根据已有文献对于各个指标意义的分析 ( Jolliffe 2000; Williams \& McCarthy ,2001 ; Weigelt \& Jolliffe 2003) ,下面我们对各个指标的结果进行解释 :

\subsubsection{1 相对产量 $(R Y)$}

对伊乐藻来说，其 $R Y$ 值在低、高两种底质营养 水平下, 皆小于 1 表明黑藻对伊乐藻种间竞争的影 响要大于伊乐藻种内竞争的影响, 即种间竞争 $>$ 种 内竞争, 方差分析表明, 密度比设置和营养对其都有 显著性影响 $(p<0.05)$ 。而对黑藻来说, 其 $R Y$ 值在 低、高两种底质营养水平下, 皆大于 1 ,表明黑藻种 内竞争的影响要大于伊乐藻对黑藻种间竞争的影 响，即种内竞争 $>$ 种间竞争，密度比设置和营养对其 没有显著性影响 $(p>0.05)$ 。

\subsubsection{2 相对产量总和( $R Y T)$}

当 $R Y T$ 等于 1 时 表明两物种实际所得产量与 期望产量相等，对环境资源有共同的需要，但种间具 体关系不明; RYT 大于 1 时 表明两物种对资源有不 同的要求, 避免竞争或成为共生关系; $R Y T$ 小于 1 时 表示两物种相互敌对。由表 2 结果可知在低、高 两种底质营养水平下, 伊乐藻、黑藻混生种群的 $R Y T$ 都小于 1 , 这说明两物种的相互关系为敌对, 存在竞 争且物种间的竞争并不平衡。

\subsubsection{3 竞争攻击力 $(A G)$}

表 3 定义的 $A G$ 是伊乐藻和黑藻相对产量的差 值, 由表可知在低、高两种底质营养水平下 $A G<0$, 
表 2 伊乐藻、黑藻实验期间生长状况

Table 2 The growth status of Elodea nuttallii and Hydrilla verticillata

\begin{tabular}{|c|c|c|c|c|c|c|}
\hline \multirow[b]{2}{*}{$\begin{array}{c}\text { 底质肥力 } \\
\text { Fertility }\end{array}$} & \multicolumn{3}{|c|}{ 伊乐藻 Elodea nuttallii } & \multicolumn{3}{|c|}{ 黑藻 Hydrilla verticillata } \\
\hline & $\begin{array}{l}\text { 最大茎长 } \\
\operatorname{MSL}(\mathrm{cm})\end{array}$ & $\begin{array}{l}\text { 茎伸长速率 }^{*} \\
\operatorname{SER}\left(\mathrm{cm} \mathrm{d}^{-1}\right)\end{array}$ & $\begin{array}{l}\text { 地下/地上 } \\
\text { Root/Shoot }\end{array}$ & $\begin{array}{l}\text { 最大茎长 } \\
\operatorname{MSL}(\mathrm{cm})\end{array}$ & $\begin{array}{l}\text { 茎伸长速率 }^{*} \\
\operatorname{SER}\left(\mathrm{cm} \mathrm{d}^{-1}\right)\end{array}$ & $\begin{array}{l}\text { 地下/地上 } \\
\text { Root/Shoot }\end{array}$ \\
\hline 低 Low & 50.75 & 0.89 & 0.022 & 121.67 & 2.12 & 0.062 \\
\hline 高 High & 62.58 & 1.39 & 0.019 & 130.24 & 2.50 & 0.026 \\
\hline
\end{tabular}

MSL : Maximum stem length ; SER : Stem elongation rate * : 茎伸长速率为黑藻茎顶端生长接近至水面时所测量的速率 The rate of stem elongation was measured when apex of the stem of Hydrilla verticillata was near the water surface

表 3 不同营养水平下的竞争指标结果

Table 3 Competition indices results at different fertility level

\begin{tabular}{|c|c|c|c|c|c|c|}
\hline \multirow{2}{*}{$\begin{array}{l}\text { 指标 } \\
\text { Indices }\end{array}$} & \multirow{2}{*}{$\begin{array}{c}\text { 营养水平 } \\
\text { Level }\end{array}$} & \multicolumn{5}{|c|}{ 密度设置 Density setup $\left(N_{\mathrm{e}}: N_{\mathrm{h}}\right)$} \\
\hline & & $1(20: 0)$ & $2(15: 5)$ & $3(10: 10)$ & $4(5: 15)$ & $5(0: 20)$ \\
\hline \multirow[t]{2}{*}{$R Y_{\mathrm{e}}$} & 低 Low & - & $0.8067 \pm 0.1356$ & $0.9203 \pm 0.1912$ & $0.5564 \pm 0.0470$ & - \\
\hline & 高 High & - & $0.6650 \pm 0.0074$ & $0.7292 \pm 0.0434$ & $0.4349 \pm 0.1032$ & - \\
\hline \multirow[t]{2}{*}{$R Y_{\mathrm{h}}$} & 低 Low & - & $1.1288 \pm 0.0351$ & $1.0216 \pm 0.1133$ & $1.0836 \pm 0.2224$ & - \\
\hline & 高 High & - & $1.0750 \pm 0.0311$ & $1.0312 \pm 0.0396$ & $1.0252 \pm 0.0713$ & - \\
\hline \multirow[t]{2}{*}{$R Y T$} & 低 Low & - & $0.9677 \pm 0.0503$ & $0.9709 \pm 0.1346$ & $0.8200 \pm 0.1284$ & - \\
\hline & 高 High & - & $0.8700 \pm 0.0188$ & $0.8802 \pm 0.0104$ & $0.7300 \pm 0.0836$ & - \\
\hline \multirow[t]{2}{*}{$A G$} & 低 Low & - & $-0.1610 \pm 0.0853$ & $-0.0510 \pm 0.0812$ & $-0.2636 \pm 0.0967$ & - \\
\hline & 高 High & - & $-0.2050 \pm 0.0126$ & $-0.1510 \pm 0.0402$ & $-0.2952 \pm 0.0296$ & - \\
\hline \multirow[t]{2}{*}{$R C I_{\mathrm{e}}$} & 低 Low & - & $0.1933 \pm 0.1356$ & $0.0797 \pm 0.1912$ & $0.4436 \pm 0.0470$ & - \\
\hline & 高 High & - & $0.3350 \pm 0.0074$ & $0.2708 \pm 0.0434$ & $0.5651 \pm 0.1032$ & - \\
\hline \multirow[t]{2}{*}{$R C I_{\mathrm{h}}$} & 低 Low & - & $-0.1288 \pm 0.0351$ & $-0.0220 \pm 0.1133$ & $-0.0840 \pm 0.2224$ & - \\
\hline & 高 High & - & $-0.0750 \pm 0.0311$ & $-0.0310 \pm 0.0396$ & $-0.0250 \pm 0.0713$ & - \\
\hline \multirow[t]{2}{*}{$R C C_{\mathrm{e}}$} & 低 Low & - & $0.5446 \pm 0.1991$ & $0.8920 \pm 0.3374$ & $0.4852 \pm 0.0479$ & - \\
\hline & 高 High & - & $0.3317 \pm 0.0073$ & $0.5750 \pm 0.0529$ & $0.3678 \pm 0.0970$ & - \\
\hline \multirow[t]{2}{*}{$R C C_{\mathrm{h}}$} & 低 Low & - & $1.1798 \pm 0.0515$ & $1.0629 \pm 0.2437$ & $1.2429 \pm 0.9559$ & - \\
\hline & 高 High & - & $1.1029 \pm 0.0438$ & $1.0668 \pm 0.0857$ & $1.1568 \pm 0.3103$ & - \\
\hline
\end{tabular}

$N_{\mathrm{e}} 、 N_{\mathrm{h}}$ 表示伊乐藻、黑藻的个体数目。表格中数值为平均值 \pm 标准差 $N_{\mathrm{e}}, N_{\mathrm{h}}$ represent the individual numbers of Elodea nuttallii and Hydrilla verticillata. The figures in the table show mean $\pm S D$

说明两物种间存在竞争, 且偏利于黑藻, 黑藻拥有相 对较强的竞争能力。方差分析表明密度设置和营养 对 $A G$ 值都有显著性的影响 $(p<0.05)$, 高营养水平 下两物种的竞争力差别较大。

\subsubsection{4 相对竞争强度 $(R C I)$}

对伊乐藻来说, 其 $R C I$ 值在低、高两种底质营 养水平下, 皆大于 0 表明受到黑藻种间竞争的影响 相对较大, 营养水平的升高对其有显著性的影响 $(p<0.05)$, 而密度比的设置对其影响也呈现出显著 性 $(p<0.05)$ 。对黑藻来说, 其 $R C I$ 值在低、高两种 底质营养水平下, 皆小于 0 ,表明受到伊乐藻种间竞 争的影响较小, 可以认为相对没有受到竞争干涉。

\subsubsection{5 相对拥挤系数 $(R C C)$}

在低、高两种底质营养水平下, 黑藻的 $R C C>$ 1 , 密度设置和营养对其没有显著性的影响 $(p>$ $0.05)$, 而伊乐藻的 $R C C<1$ 密度设置和营养对其有
显著性的影响 $(p<0.05)$, 表明黑藻的竞争能力要强 于伊乐藻。

综合以上各个指标的结果, 可以看出在低、高两 种底质营养水平下黑藻的竞争能力都要强于伊乐 藻, 且在高营养水平下, 黑藻的竞争能力有所增强。

\subsection{3 取代系列实验图表模型}

根据实验所得混合种植时每盆伊乐藻、黑藻实 测生物量干重, 以及依据单物种种植时伊乐藻、黑藻 各自生物量干重按照密度比例设置各自在混合种植 时所预期应得到的干重，绘制取代系列实验图表如 下：

由图 2,3 可以看出，组成种对混生种群总生物 量的贡献值并不相同，与其各自的播种比例不是成 正比例的关系, 其中伊乐藻对混生种群生物量的贡 献值比预期值小, 而黑藻对混生种群生物量的贡献 值要比预期值大, 且混生种群的总生物量比单种种 


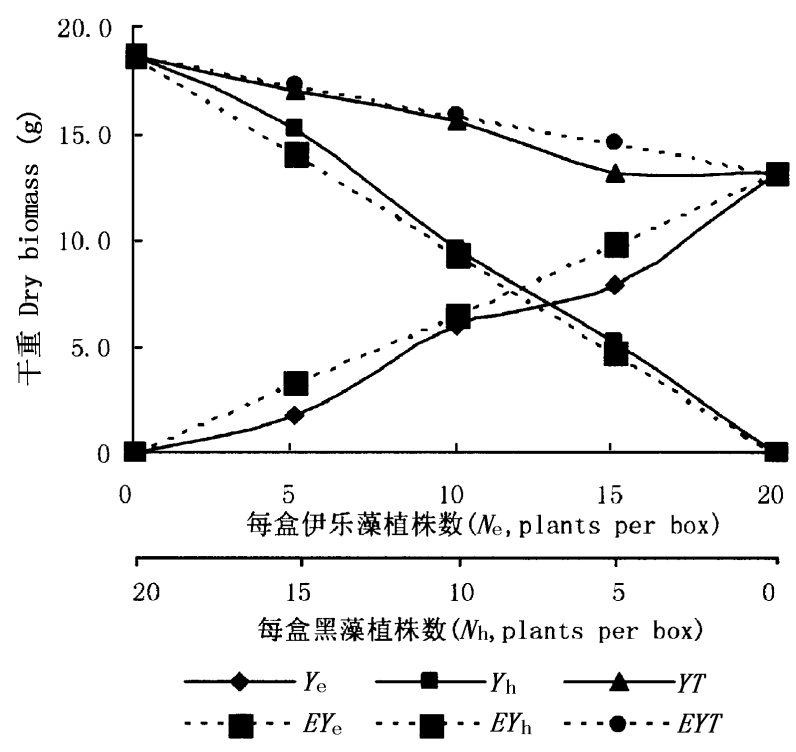

图 2 低营养底质下的取代系列实验图表

Fig.2 Replacement diagram at low fertility

$Y_{\mathrm{e}} 、 Y_{\mathrm{h}} 、 Y T 、 E Y_{\mathrm{e}} 、 E Y_{\mathrm{h}} 、 E Y T$ 分别代表伊乐藻、黑藻实测干重以及 两者总干重和按照种植密度比例预期应得到的伊乐藻、黑藻干重以 及两者总干重。预期值是指在理想状态下, 即物种种内竞争 $=$ 种间 竞争的前提下, 按照密度比例依据单种生物量所得到的值 $Y_{\mathrm{e}}, Y_{\mathrm{h}}$, $Y T$ respectively represent the observed mean dry biomass of Elodea nuttallii, Hydrilla verticillata and the total dry biomass in the experiment, whereas $E Y_{\mathrm{e}}, E Y_{\mathrm{h}}, E Y T$ respectively represent the expected dry biomass of Elodea nuttallii , Hydrilla verticillata and the total dry biomass by virtue of the density ratios according to monoculture. Here, the expected dry biomass is obtained when inter- and intra- specific interference are equal

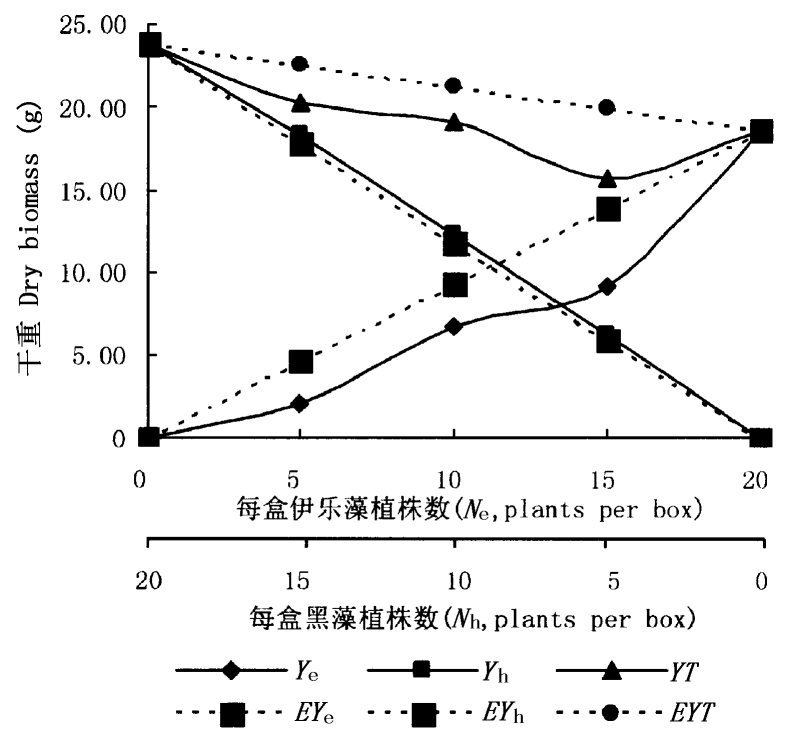

图 3 高营养底质下的取代系列实验图表

Fig.3 Replacement diagram at high fertility 图注同图 2 Notes see Fig. 2
群的生物量低，即其总生物量小于预期值，这表示两 物种之间的相互关系为相互敌对, 黑藻对伊乐藻生 活环境的不利影响要大于其对自身的影响，即种间 竞争强度大于种内竞争强度, 从而抑制了伊乐藻的 生长。这种相互关系在两种底质营养水平下类同， 都表现出同样的趋势。

为考查物种的竞争能力，我们结合产量 - 密度 方程, 进一步说明取代系列实验图表的结果。在低 营养底质水平下, 得到方程如下：

$$
\begin{aligned}
& Y_{\mathrm{e}}=N_{\mathrm{e}} /\left(0.31 \times 10^{-2}+0.0767 N_{\mathrm{e}}+0.1173 N_{\mathrm{h}}\right) \\
& R^{2}=0.942 \quad N=15 \\
& Y_{\mathrm{h}}=N_{\mathrm{h}} /\left(0.16 \times 10^{-2}+0.0527 N_{\mathrm{h}}+\right. \\
& \left.0.0465 N_{\mathrm{e}}\right) R^{2}=0.925 \quad N=15
\end{aligned}
$$

对于伊乐藻 (方程 3 ), 种内竞争与种间竞争系 数的比值为 $A_{\mathrm{ee}} / A_{\mathrm{eh}}=0.0767 / 0.1173=0.654$, 表明 在混合种植情况下来自黑藻的种间竞争要比来自其 它伊乐藻植株的种内竞争重要的多。增加一株伊乐 藻和增加 0.654 株黑藻对伊乐藻平均生物量的影响 是相同的, 也就是说在低营养下, 一株伊乐藻与 0.654 株黑藻竞争力相等。而对于黑藻 (方程 4) , 种 内竞争与种间竞争系数的比值为 $A_{\mathrm{hh}} / A_{\mathrm{he}}=$ $0.0527 / 0.0465=1.133$,表明一株黑藻与 1.133 株 伊乐藻对混合种植下黑藻平均生物量的影响相等。

在高营养底质水平下, 得到方程如下：

$$
Y_{\mathrm{e}}=N_{\mathrm{e}} /\left(0.25 \times 10^{-2}+0.0540 N_{\mathrm{e}}+0.1280 N_{\mathrm{h}}\right)
$$

$R^{2}=0.949 \quad N=15$

$Y_{\mathrm{h}}=N_{\mathrm{h}} /\left(0.88 \times 10^{-2}+0.0415 N_{\mathrm{h}}+\right.$

$\left.0.0382 N_{\mathrm{e}}\right) R^{2}=0.990 \quad N=15$

对于伊乐藻 (方程 5 ), 种内竞争与种间竞争系 数的比值为 $A_{\mathrm{ee}} / A_{\mathrm{eh}}=0.0540 / 0.1280=0.422$, 表明 一株伊乐藻与 0.422 株黑藻对混合种植下伊乐藻平 均生物量的影响相等。而对于黑藻来说(方程 6), 种内竞争与种间竞争系数的比值为 $A_{\mathrm{hh}} / A_{\mathrm{he}}=$ $0.0415 / 0.0382=1.086$, 即一株黑藻与 1.086 株伊 乐藻对混合种植下黑藻平均生物量的影响相等。

综合实验图表以及拟合方程的结果，可以看出 黑藻具有比伊乐藻强的竞争能力, 且随底质营养的 升高 种间竞争能力有所增强。

\section{2 黑藻、伊乐藻周年竞争态势}

黑藻和伊乐藻都具有较强的断枝繁殖能力, 栽 植 $3 \mathrm{~d}$ 后, 基本所有的植株都生出不定根, 植株成活 率较高, 达到 $100 \%$ 。黑藻的茎生长速率较快, 种植 $15 \mathrm{~d}$ 左右即可生长至水面, 此后茎端在水面延伸生 
长, 伊乐藻生长稍慢, 种植 $20 \mathrm{~d}$ 左右也可生长至水 面, 但始终在水面以下生长, 属完全沉水。种植 1 个 月后, 伊乐藻、黑藻植株生长旺盛, 产生大量分枝, 覆 盖满整个水缸水面, 在所有重复中 (单种种植除外), 没有明显取代现象发生, 伊乐藻、黑藻皆能正常生 长, 10 月中下旬, 黑藻基本达到最大生长, 在水面产 生较多的断枝，但由于伊乐藻在近水面处产生较多 的分枝, 且枝条纤细, 形成茂密的冠层, 导致大多数 黑藻断枝不能沉入水底而开始腐烂，只有极少数的 断枝可以沉水。秋末冬初 11 月 10 日, 黑藻完成其 生活史, 大部分枝条腐烂, 直立茎尖端产生的少量鳞 状芽苞即冬芽脱离枝条, 由于重力的作用沉入水底, 准备过冬, 而此时伊乐藻仍然具有较强的生长活力, 继续生长, 在黑藻腐烂后, 迅速占领整个水体。约在 12 月 15 日左右, 由于最低气温降至 $0{ }^{\circ} \mathrm{C}$ 以下, 伊乐 藻生长明显减缓甚或停滞生长, 新枝条萌生数量减 少, 水体上层枝条由于水温降低而衰败腐烂,下部枝 条仍然存在, 并能维持活力顺利越冬, 即使在水面有 薄冰出现时仍可见有嫩枝形成。

来年春季随着气温的回升 2005 年 3 月 15 日观 察时, 伊乐藻已经开始萌发出新的芽体, 并有少量嫩 枝形成，而黑藻没有任何动静。5 月 1 日观察时,伊 乐藻生长旺盛, 主茎高度超过 $30 \mathrm{~cm}$, 黑藻单种种植 的缸内萌发出新的幼苗 取样发现, 大部分的幼苗都 是由基部褐色或黑褐色的冬芽萌发产生，其余少量 则是由埋在泥中的匍匐茎的腋芽或顶芽萌发产生 的，而在混合种植的缸内，未见有任何的黑藻植株萌 发。5 月 29 日观察时发现, 除在黑藻与伊乐藻密度 比为 $25: 5$ 的处理内有 $2 \sim 3$ 株黑藻存在外, 其它处 理中未见任何的黑藻植株萌发，此后一段时间直至 实验结束, 也没有新的黑藻植株萌发, 水缸全部为伊 乐藻所占领。

\section{3 讨论与结论}

在水生生境中, 最激烈的竞争常常容易发生在 具有相似生长型占据水体相同位置的物种间 ( Gopal \& Goel ,1993)。伊乐藻和黑藻都为大型沉水植物， 在水体中的位置有较大的重叠, 且两者的营养体形 态和生长方式极为相似, 都拥有较强的分枝能力, 生 物量的大部分都分布在植株的中上部, 形成类似于 树冠一样的冠层, 并且两者都拥有较强的无性繁殖 能力, 因此当两种植物生活在同一混合群落中时 就 难免发生竞争。

自然环境条件下，植物生长受到诸多生物和非
生物因素的作用，要研究植物间的相互作用便受到 较多逻辑和分析计算上的限制。因此, 常常通过构 建人工植物群落的方法, 在人为控制条件下进行研 究。同自然状态相比, 人为控制环境条件下可能存 在生境(如水体)较小、混合群落规模小等局限, 但它 可以研究植物在特定的年龄阶段或个体发育的不同 时期及在不同的专一控制环境条件下的种间相互作 用，更容易的评估其它因素的影响，并能对影响竞争 的物种生物学特征做出较为准确的判断，从而可以 为预测植物在野外的竞争力提供有力的参考。

在本实验条件下,从短期来看，在黑藻、伊乐藻 同处于旺盛生长季节时, 黑藻的竞争能力要强于伊 乐藻, 且竞争能力随底质肥力升高而增强, 对黑藻来 说其种内竞争的影响要大于伊乐藻对它的种间竞争 影响。我们认为这主要是与其以下生物学特性有 关 :其一黑藻和伊乐藻在个体大小上具有明显的差 异。黑藻的个体较大, 植株抽条直径一般在 $3 \sim 5$ $\mathrm{mm}$ 左右, 而伊乐藻的抽条直径略小, 仅在 $1 \sim 2 \mathrm{~mm}$ 左右, 此外, 黑藻的茎生长速率较快, 在低高两种底 质营养下, 初期伸长速率为 2.12 和 $2.50 \mathrm{~cm} \mathrm{~d}^{-1}$, 而 伊乐藻的茎生长速率则较慢, 伸长速率仅为 0.89 和 $1.39 \mathrm{~cm} \mathrm{~d}^{-1}$, 在实验期间黑藻的主茎高度达到伊乐 藻的 2 倍多。因此 断枝扞插后, 黑藻能够较早的长 至水面, 在水面上弯曲漂浮生长。其二, 尽管伊乐 藻、黑藻都有冠层 (Canopy) 的形成, 但两者冠层并不 是完全相同。黑藻植株可以长出水面, 在水面处形 成茂密的上冠层 (Upper canopy), 而伊乐藻的冠层为 位置相对较下的下冠层 (Lower canopy) ,处于近水面 以下的位置, 因此黑藻对伊乐藻造成了一定的遮荫 作用，在阳光的获取上占据优势。有无冠层的形成 在沉水植物竞争中起到重要的作用 (Barko et al. , 1986) ,例如 Moen 和 Cohen(1989) 在研究篦齿眼子菜 (Potamogeton pectinatus) 和狐尾藻 (Myriophyllum excalbescens) 的竞争中发现，篦齿眼子菜在水面形成的 茂密冠层，对狐尾藻造成了遮荫作用，抑制了狐尾藻 的生长, 而后者仅仅在近水面下有叶漂浮生长。V V a 等(1999)也曾在对黑藻和美洲苦草(Vallisneria americana) 竞争研究中发现, 土壤肥力丰富下黑藻拥有强 的竞争能力就是因为黑藻能够分配约 $95 \%$ 的生物 量在光合作用组织，在近水面处形成了茂密的冠层， 因此在阳光的获取上具有较明显的优势。Hofstra 等 (1999) 在对黑藻在新西兰的竞争表现研究中也发 现, 黑藻的高位冠层是其比其它沉水植物表现出竞 争优势的一大因素。其三, 黑藻的根系较伊乐藻发 
达, 在底质营养的吸收上可能占有优势。虽然两者 的根系都为不发达的不定根根系, 但黑藻在植株茎 基和匍匐茎的节部都能产生较伊乐藻数量多的不定 根, 造成在同样底质条件下, 黑藻的根冠比要高于伊 乐藻(表 2), 而根系在两者的营养吸收上都具有较 大作用, 故黑藻根系可能在营养吸收上占有优势。

从周年实验结果来看，尽管在旺盛生长期，黑藻 具有一定的优势, 例如植株个体较大; 具有较快的茎 伸长速率; 可以在水面生长, 更好的利用阳光等, 但 经过一个冬季后, 伊乐藻反而在竞争中占有了绝对 的优势地位, 之所以会产生这样的结果, 我们认为原 因可以主要从温度适应和繁殖方式方面讨论 :温度 适应方面，伊乐藻属于冬春季优势物种，比较耐寒， 当 11 月黑藻植株枯萎后, 伊乐藻还可以继续生长, 填补占领黑藻死亡后留下的空间, 在冬季水温高于 $4 \pm 1{ }^{\circ} \mathrm{C}$ 时 植株仍然可以继续保持活力状态正常生 长（Kunii，1981,1982,1984），维持其种群规模，甚至 当水温低至 $0{ }^{\circ} \mathrm{C}$ 时, 仍可保持生长活力, 在温度适宜 时, 表现出多年生植物的生长特性。繁殖方式方面， 伊乐藻不产生任何特殊的营养繁殖体, 当冬季水温 降低后, 主要以无固定的具叶的短枝形式在水底形 成密集的枝丛垫层 (Dense mat) 越冬, 当春季 (3 月中 下旬) 水温高于 $10{ }^{\circ} \mathrm{C}$ 时即可有新的植株由匍匐枝的 芽体萌发, 新枝的伸长速率较快, 达到 $3 \mathrm{~cm} \mathrm{~d}^{-1}(\mathrm{Ku}-$ nii ,1984)。而黑藻喜温耐热, 进入冬季后, 植物体腐 败, 不能越冬 种群再生主要依赖于特殊营养繁殖体 冬芽, 以及一部分匍匐茎上面膨大的顶芽, 而冬 芽的休眠期较长，萌发需要一定的光照和水温条件， 一般在 3 月中下旬才可萌发, 而新枝条的萌生和伸 长要在 4 月上旬, 且初始生长缓慢, 两昼夜仅增长 3 $\mathrm{mm}$ 至 4 月中下旬，生长才逐渐加快(文明等, 1994, 1995 陈洪达,1989），而此时伊乐藻已经处于旺盛生 长期, 形成了比较茂密的群丛, 因此对黑藻繁殖体的 萌发和幼苗的生长造成了一定的抑制作用，导致黑 藻不能正常的萌发。我们在实验时也发现, 即便在 没有伊乐藻的存在下, 黑藻新植株的萌发也要发生 在 4 月底，因而，伊乐藻在冬春时间和空间的竞争中 占有明显的优势, 这是其之所以竞争成功的关键所 在。Kunni (1984) 在对伊乐藻季节生长及形态构建 的研究中也认为伊乐藻的耐寒性、营养体的越冬方 式是使其在同其它植物的生长中占有优势, 而胡耀 辉 (1996) 所做的几种沉水植物伊乐藻、黑藻、金鱼 藻、微齿眼子菜的竞争态势试验也发现, 在当年的生 长中伊乐藻、黑藻都生长良好, 未发现有明显的取代
和界面侵入现象发生, 而经过一次生长期和休眠期 后的 1995 年, 黑藻就几乎不存在了, 他认为伊乐藻 在冬春时空竞争方面占有优势, 当早春黑藻处于休 眠期时，伊乐藻已处于生长期。这与我们的实验结 果基本吻合。因此, 如果应用黑藻进行水生植被恢 复工作, 需要在种植时间上有所考虑, 同时应该与不 同生长季节的水生植物互相补充, 例如菹草 (Potamogeton crispus) 最大限度地保证水体周年均有水生 植物生长。这些植物如果在秋冬季节对伊乐藻存在 竞争优势，即可利用这些植物的搭配实现水生植物 的恢复重建, 同时限制伊乐藻的扩张, 这些工作需要 进一步开展。

影响竞争结果的因素有很多, 主要的因素有种 间差异、菌根真菌、寄生生物、草食性动物、幼苗出土 时间、竞争期的长短、营养有效性、空间分布的斑块 性等等(李博,2001)。这些因素并不是孤立存在的， 通常往往是几种甚至是多种因素掺杂在一起，共同 来影响物种间的竞争。另外,由于竞争能力是植物 的综合能力，它应该由植物的形态、生理及生活史特 征共同决定 (Aarssen,1983) 单个植物特征不可能解 释所有物种竞争能力的差异, 因此要全面准确的了 解物种间的竞争关系, 必须对其特征有着全面的了 解。我们的实验仅是在人工模拟条件下，通过物种 间的竞争表现，对影响伊乐藻、黑藻竞争关系的生物 学特征做了一些初步的探讨,希望能够为其种间关 系的研究提供一定的参考, 而在自然状态下 植物间 的竞争关系要比人为设计复杂得多, 这方面的实验 工作也还有待深入。

\section{参 考 文 献}

Arassen LW (1983) . Ecological combining ability and competitive combining ability in plants: toward a general evolutionary theory of coexistence in systems of competition. American Naturalist, $122,707-731$.

Barko JW, Adams MS, Clesceri NL (1986). Environmental factors and their consideration in the management of submersed aquatic vegetation: a review. Journal of Aquatic Plant Management, 24, $1-10$.

Barrat-Segretain MH (2001). Invasive species in the Rhone River floodplain (France) : replacement of Elodea canadensis Michaux by $E$. nuttallii St. John in two former river channels. Archiv Fur Hydrobiologie, 152, $237-251$.

Barrat-Segretain MH, Elger A, Sagnes P, Puijalon S (2002) . Comparison of three life-history traits of invasive Elodea canadensis Michx. and Elodea nuttallii (Planch.) H. St. John. Aquatic Botany, 74, 299 - 313 . 
Baumann DT, Bastiaans L, Kroff MJ (2001) . Competition and crop performance in a leek-celery intercropping system. Crop Science, $41,764-774$.

Best EPH, Dassen JHA (1987) . A seasonal study of growth characteristics and the levels of carbohydrates and proteins in Elodea nuttallii, Polygonum amphibium and Phragmites australis. Aquatic Botany, 28, 353 - 372 .

Chen HD（陈洪达）（1989）. Studies on productivity of Hydrilla verticillata Royle. Journal of Wuhan Botanical Research (武汉植 物学研究), 7, 77-85. (in Chinese with English abstract)

Cousens R (1991). Aspects of the design and interpretation of competition (interference) experiments. Weed Technology, 5, $664-$ 673.

Cui XH (崔心红), Xiong BH (熊秉红), Pu YH (蒲云海), Li W (李伟), Chen JK (陈家宽), He GQ (何国庆) (2000). Comparative study of regeneration and colonization ability in five submersed macrophytes. Acta Phytoecologica Sinica (植物生态 学报), 24, 502-505. (in Chinese with English abstract)

de Wit CT (1960) . On competition. Verslagen van Landbouwkundige Onderzoekingen, 66, 1-82.

dt Wit CT, van den Bergh JP (1965) . Competition between herbage plants. Netherlands Journal of Agricultural Science, 13, 212 221 .

Firbank LG, Watkinson AR (1985). On the analysis of competition within two-species mixtures of plants. Journal of Applied Ecolo$g y, 22,503-517$.

Fowler N (1982) . Competition and coexistence in a North Carolina grassland. III. Mixture of component species. Journal of Ecology, 70, $77-92$.

Freckleton RP, Watkinson AR (1999). The mis-measurment of plant competition. Functional Ecology, 13, 285 - 287.

Gao G (高光) (1996). Experimental studies on the effect of purification of fishculture waste water by Elodea nuttallii and Hydrilla verticillata. Journal of Lake Sciences (湖泊科学), 8, 184 188. (in Chinese with English abstract)

Gaudet CL, Keddy PA (1988). A comparative approach to predicting competitive ability from plant traits. Nature, 334, $243-$ 244 .

Gibson DJ, Connolly J, Hartnett DC, Weidenhamer JD (1999) . Designs for greenhouse of interactions between plants. Journal of Ecology, 87, 1 - 16 .

Gopal B, Goel U (1993). Competition and allelopathy in aquatic plant communities. Botanical Review, 59(3), $155-210$.

Grace JB (1995) . On the measurement of plant competition intensity. Ecology, 76, $305-308$.

Grime JP (2001). Plant Strategies, Vegetation Processes and Ecosystem Properties 2nd edn. Wiley, Chichester, London, 1.

$\mathrm{Gu}$ XH (谷孝鸿), Zhang SZ (张圣照), Bai XL (白秀玲), $\mathrm{Hu}$ WP (胡维平), $\mathrm{Hu} \mathrm{YH}$ (胡耀辉), Wang XR (王晓蓉) (2005) . Evolution of community structure of aquatic macrophytes in East Taihu Lake and its wetlands. Acta Ecological Sinica (生
态学报), 25, 1541 - 1548. (in Chinese with English abstract) Hofstra DE, Clayton J, Green JD, Auger M (1999). Competitive performance of Hydrilla verticillata in New Zealand. Aquatic Botany, 63, 305-324.

$\mathrm{Hu}$ YH (胡耀辉) (1996). Testing study of biomass, production and competitive growth of Elodea nuttallii and other submerged plants. Journal of Lake Sciences (湖泊科学), 8 (Suppl. ), 73 - 78. (in Chinese with English abstract)

Jolliffe PA (2000). The replacement series. Journal of Ecology, $88,371-385$.

Kunii H (1981). Characteristics of the winter growth of detached Elodea nuttallii (Planch.) ST. John in Japan. Aquatic Botany, $11,57-66$.

Kunii H (1982). The critical water temperature for the active growth of Elodea nuttallii (Planch.) St. John. Japanese Journal of Ecology, 32, 111 - 112 .

Kunii H (1984). Seasonal growth and profile structure development of Elodea nuttallii (Planch.) St. John in pond Ojaga-Ike, Japan. Aquatic Botany, 18, 239-247.

Lian GH (连光华), Zhang SZ (张圣照) (1996). Artificial vegetative reproduction and plantation technique for Elodea nuttallii and six other species of submerged plants. Journal of Lake Sciences (湖泊科学) , 8(Suppl.), 11 - 16. (in Chinese with English abstract)

Li B (李博) (2001). Plant Competition (植物竞争——作物与 杂草相互作用的实验研究). Higher Education Press, Beijing; Springer Press, Heidelberg, 42 - 64. (in Chinese)

Li B, Hara T (1999). On the relative yield of plants in a twospecies mixture. Oikos, 85, 170-176.

McGilchrist CA, Trenbath BR (1971). A revised analysis of plant competition experiments. Biometrics, 27, 659-671.

Moen RA, Cohen Y (1989). Growth and competition between Potamogeton pectinatus L. and Myriophyllum excalbescens Fern. in experimental ecosystems. Aquatic Botany, 33, 257-270.

Spitters CJT (1983). An alternative approach to the analysis of mixed cropping experiments. 1. Estimation of competition effects. Netherlands Journal of Agricultural Sciences, 31, 1-11.

Tilman D (1988). Plant Strategies and Dynamics and Structure of Plant Communities . Princeton University Press, Princeton, 1.

Turkington R, Jolliffe PA (1996). Interference in Trifolium repensLolium perenne mixtures: short- and long-term relationships. Journal of Ecology, 84, 563 - 571 .

Van TK, Wheeler GS, Center TD (1999). Competition between Hydrilla verticillata and Vallisneria americana as influenced by soil fertility. Aquatic Botany, 62, 225-233.

Watkinson AR (1981). Interference on pure and mixed populations of Agrostemma githago. Journal of Applied Ecology, 18, 967 976.

Weigelt A, Jolliffe P (2003). Indices of plant competition. Journal of Ecology, 91, 707 - 720 .

Wen M (文明), Sheng Z (盛哲), Lin QZ (林亲众) (1994)。 
Study of Hydrilla verticillata (L.f.) royle as protein resource. I . Analysis of the biological characters and nutrition elements of Hydrilla verticillata (L.f.) royle. Journal of Hunan Agricultural College (湖南农学院学报), 20,457-463. (in Chinese with English abstract)

Wen M (文明), Sheng Z (盛哲), Lin QZ (林亲众) (1995). Study of Hydrilla verticillata (L.f.) royle as protein resource. II . Study of the quality of ecology and the experiment of introduction and cultivation of this plant. Journal of Hunan Agricultural College (湖南农学院学报), 21, 10-16. (in Chinese with English abstract)

Williams AC, McCarthy BC (2001). A new index of interspecific competition for replacement and additive designs. Ecological Journal, 16, $29-40$.

Wright AJ (1981). The analysis of yield-density relationships in binary mixtures using inverse polynomials. Journal of Agricultural Sciences, 96, $561-567$.
Wuhan Institute of Botany, Chinese Academy of Sciences (中国科 学院武汉植物研究所) (1983). Picture of Aquatic Vascular Plants in China (中国水生微管束植物图谱). Hubei People's Press, Wuhan, 114. (in Chinese)

Zhu SQ (朱松泉), Liu ZW (刘正文) (1996). Study on establishing an artificial compound ecosystem of Elodea-grass carp pen farming. Journal of Lake Sciences (湖泊科学), 8 (Suppl.), 46-61. (in Chinese with English abstract)

Zhang SZ (张圣照), Wang GX (王国祥), Pu PM (兴培民), Chigira T (千金良-达哉)（1999）. Succession of hydrophytic vegetation and swampy tendency in East Taihu Lake. Journal of Plant Resource and Environment (植物资源与环境), 8(2), 1-6. (in Chinese with English abstract)

Zhu W (朱伟), Chen QJ (陈清锦), Zhang LF (张兰芳) (2004). Purification effect of polluted water in low temperature in winter by Elodea nuttallii. Ecology and Environment (生态环 境) , 13, 497 - 499. (in Chinese with English abstract)

责任编委 :于 丹 责任编辑 :刘丽娟 